\title{
Measurement of intimacy: conceptual and methodological issues of studying close relationships ${ }^{1}$
}

In the broad context, an understanding of the role of intimacy has been discussed as essential to the development of a science of interpersonal relationships (Hinde, 1978). Specifically, the absence of an intimate confiding relationship has been identified as a vulnerability factor in the onset of depression under adverse circumstances (Brown \& Harris, 1978 $a$; Costello, 1982; Solomon \& Bromet, 1982). Aneshensel \& Stone (1982) suggest that, assuming that lack of perceived social support is not just a manifestation of depression itself, lack of intimacy may contribute to the creation of depressive symptoms independent of life events. Henderson et al. (1981) suggest that the perceived availability and adequacy with which significant others meet an individual's requirements for attachment play a small but significant role in the onset of non-psychotic emotional illness under adverse circumstances. Waring et al. $(1981 a)$ found that deficiencies of marital intimacy were significantly associated with the presence of symptoms of non-psychotic emotional illness in one or both spouses. The significance of these important studies is dependent on the reliability and validity of the methods of measuring intimacy and the conceptual definition of intimacy.

Several conceptual and methodological issues raised by these studies merit a closer examination. The studies described above have largely employed structured sociological interviews, containing questions about the quality of personal relationships. These questions allow a rating of the depth of 'intimacy' by a trained interviewer.

One question which arises about the concept of intimacy evaluated in these interviews is: "Whose intimacy is being measured? Hers? His? Or theirs?' The possibility exists that the perception of relationships as unavailable or inadequate might be a product of an individual's attitudes or moods. On the other hand, they might be a valid expression of how others have behaved towards the respondent. Data which record the spouse's or partner's perceptions of the relationship might be essential to evaluate the theoretical possibility that perceived differences in intimacy may have a different impact on vulnerability to emotional illness from congruent perception.

A second question is whether narrow operational definitions of intimacy or broad definitions of the concept are to be preferred (Schaefer \& Olson, 1981). Several authors have addressed this second conceptual and methodological question (Tennant \& Bebbington, 1978; Tennant et al. 1982; Shapiro, 1979). They assert that the variables of marital status and the quality of the marital relationships have been confounded. Brown \& Harris $(1978 b)$ reply that, although these variables are interlinked, they are not confounded: many unmarried women had intimate relationships with their boyfriends and many married women were unable to confide in their husbands. Separate analysis of the data for the unmarried and the married might be instructive.

Thirdly, these sociological measures involved no discussion as to whether scores for partners should be analysed separately or as joint scores on their combined level of interpersonal intimacy in comparison with general population means or standards.

Finally, recent evidence suggests that intimacy can be defined as a multifaceted dimension of interpersonal relationships which may or may not have been defined too narrowly by several questions regarding confiding in a spouse and availability and/or adequacy of close relationships (Schaefer \& Olson, 1981; Waring et al. 1980). Recent studies suggest that a stronger correlation between depression and deficiencies of marital intimacy than that originally reported by Brown \& Harris may be found by broader definitions of intimacy (Waring \& Patton, 1984). Although the

\footnotetext{
${ }^{1}$ Address for correspondence: Dr E. M. Waring, Department of Psychiatry, Victoria Hospital, 375 South Street, London, Ontario N6A 4G5, Canada.
} 
above questions merit closer scrutiny, various replications of these studies of intimacy and symptoms of non-psychotic emotional illness suggest that further research is merited.

This review will focus on recent methods developed to measure intimacy and will discuss the reliability and validity of three different techniques: (1) sociological surveys, (2) self-report questionnaires, and (3) structured interviews. These methods are designed to measure the quality and quantity of $(a)$ social, $(b)$ interpersonal, and $(c)$ marital intimacy. The observations may provide some insight into the methodological complexity of measuring intimacy in interpersonal relationships.

\section{MEASUREMENT OF SOCIAL INTIMACY}

The pioneering work of Brown \& Harris (1978a), suggesting that lack of intimacy might be a vulnerability factor in the development of depression in women under adverse circumstances, has been widely cited and replicated (Solomon \& Bromet, 1982; Costello, 1982). The authors examined the protective role of more enduring relationships by interviewing women and asking them to name people to whom they could talk about things that were troubling them. Their rating of the quality of relationships depended mainly on replies to these questions.

The standard questions on the Interview Schedules for Life Events and Difficulties were: (i) If you had a problem of some sort, who would be the first person you would want to discuss it with? (ii) What about your... husband [pause], mother [pause, etc.], sister, father, brother? What about any friends? Are any of them close enough for you to confide in? (iii) Is your husband easy to talk to in general about things? Does he get bored or stop listening? Or say that women are always worrying about things that are not really important?

Face validity suggests that these questions are evaluating only one characteristic of intimacy self-disclosure. While self-disclosure is a major covariate of intimacy, the two constructs are not equivalent (Waring \& Chelune, 1983). Whether a narrow definition of interpersonal intimacy or a multifaceted definition is to be preferred must await further research. The possibility exists that the relationship between emotional illness and intimacy may be strengthened or weakened by different operational definitions.

Women on the high point of the scale, ' $a$ ', were considered likely to have a close, intimate, and confiding relationship with a husband or boyfriend; those on levels ' $b$ ' and ' $c$ ' had other confidants; and those on level ' $d$ ' had none. Of the 458 women studied in Camberwell, $63 \%$ were rated ' $a$ ', $22 \%$ were rated ' $b$ ', $8 \%$ were rated ' $c$ ', and $6 \%$ were rated ' $d$ '. In the presence of a provoking agent, one in ten of ' $a$ ' subjects, one in four of ' $b$ ' subjects, and one in every 2.5 of ' $c$ ' and ' $d$ ' subjects developed depression. The fact that $63 \%$ of women had a confidant is extremely high, considering a recent study that demonstrated that only $18 \%$ of a general population sample of married couples had optimal intimacy, using a broader definition of marital intimacy (Waring, 1983).

One might also wonder about the accuracy of the data, as the ratings are based only on the respondent's perception of a relationship. Depressed women may perceive relationships negatively (Beck, 1963). The confidant's perception of the relationship is not included in the data analysis. A recent study suggests that perception of deficiencies of intimacy in marriage is not simply a result of the depressed state, but that the perception of spouses do differ about the quality of the relationship (Waring \& Patton, 1984).

Henderson et al. (1981) have conducted a similar epidemiological study of the relationship of neurosis to the social environment. They have developed the Interview Schedule for Social Interaction (ISSI), which measures the availability and adequacy of attachment and social integration. These authors provide data on the ISSI's reliability indices which are acceptable for internal consistency using Cronbach's alpha and split-half coefficients. The test-retest reliability indices range from 0.51 to 0.76 for an 18-day interval and are not impressive. The authors present data suggesting that the measurement of availability of attachment and social integration is very stable over time, 'like a personality trait'. Measures of the adequacy of attachment show a greater fluctuation. 
The authors' data regarding validity are less convincing, for conceptual and methodological reasons. First, they argue the face validity of the questions, which include: (1) Is there any particular person on whom you feel you can lean? (2) Do you feel that there is one particular person who feels very close to you? (3) At present, do you have someone with whom you can share your most private feelings with (confide in) or not? (4) Recently, have you been having any unpleasantness or rows with anyone close to you?

These questions raise two conceptual problems: the questions define intimacy very narrowly in terms of self-disclosure; and they are not specific for any one relationship. The authors note that they also tap a stable quality of perception of relationships which may well be a personality trait or attitude, rather than the actual quality of a relationship.

A second question deals with the truth or accuracy of the responses to the questions. The authors studied a criterion group of recent arrivals in Canberra and found validity for social integration, but a question remains about the accuracy of their measurement of attachment. In fact, they found a correlation between high neuroticism and low adequacy and availability of attachment, suggesting the possibility that they are measuring neuroticism or, as they state, 'anxious attachment'.

Henderson et al. (1981) do make the important methodological step of obtaining information about the respondent from another person. Half the respondents in their fourth evaluation named a person who could be interviewed. The correlations between respondent and informant were: availability of attachment (AVAT), 0.42 ; adequacy of attachment (ADAT), 0.39 ; availability of social integration (AVSI), 0.59 ; adequacy of social integration (ADSI), $0 \cdot 26$. Although these values are all significant, they are considerably below the correlation of 0.60 between spouses on some measures of marital adjustment (Dean \& Lucas, 1978). This suggests that the accuracy of perception between partners regarding AVAT and ADAT, the measures of intimacy, may be questioned (Waring et al. 1984). The possibility exists that relationships in which one partner perceives a lack of intimacy which the other partner does not acknowledge may have a different relationship to symptoms from those relationships where both partners' perceptions are congruent.

Recently, Miller \& Lefcourt (1982) have developed a 17-item self-report questionnaire to measure social intimacy. The Miller Social Intimacy Scale (MSIS) measures the maximum level of intimacy currently experienced. Internal consistency, test-retest reliability and convergent, discriminant and construct validity are discussed. The development sample $(N=252)$ consists of 188 undergraduate students, 17 married student couples, and 15 couples seeking conjoint marital therapy. An initial item pool $(N=30)$ was developed atheoretically from systematic interviews with 50 undergraduate students which explored the nature of their relationships. The authors developed a 10-point frequency and intensity scale. The questions tap frequency of confiding, expression of affection, disagreement, feelings of closeness, and the importance of the relationship. The reliability, using Cronbach's alpha $(0.91)$ and test-retest reliability $(0.96)$, suggests stability. Convergent validity with measures of trust, intimacy, and loneliness were quite high, but the psychometric qualities of these criterion tests were not reported. The intimacy scores were higher for the married students than for the unmarried.

The major difficulty with the questionnaire method is whose social intimacy is being measured hers, his or theirs? No correlations of intimacy scores between the normal or disturbed married couples is presented. Studies of marital intimacy scores for couples usually report that scores correlate about 0.60 (Waring et al. 1983). Dean \& Lucas (1978) have examined the issue of whether one uses an individual's or a couple's score. They found correlations between a measure of marital adjustment and a measure of communication to be 0.73 for individual scores, 0.73 for minimum couple's score, 0.71 for maximum couple's score, and 0.76 for the mean. In spite of very plausible theoretical concerns, the issue of whose intimacy is being measured did not appear to be of practical importance in their study. On the other hand, differences in the perception of social intimacy may be more relevant to issues of emotional illness than mean scores. In summary, this self-report questionnaire utilizes a broader operational definition of intimacy, but issues of validity remain unresolved. This questionnaire has not been used to study the prevalence of emotional illness in the general population. 


\section{THE MEASUREMENT OF INTERPERSONAL RELATIONSHIPS}

Schaefer \& Olson (1981) have developed the Personal Assessment of Intimacy in Relationships (PAIR), which provides information on five types of intimacy: emotional, social, sexual, intellectual, and recreational. Individuals, married or unmarried, describe their relationship in terms of how they currently perceive it (perceived) and how they would like it to be (expected).

The operational definition of intimacy was developed from a review of the literature, and concepts were derived from family therapists, mental health professionals, and lay persons. One hundred and thirteen items selected from a pool of 350 items for conceptual clarity and appropriateness to category were given to a pilot sample of 85 people, the majority of whom were students. Cronbach's alpha reliability coefficients were above $\mathbf{0 . 7 0}$ for all six scales, including a conventionality scale. Criterion validity with measures of marital adjustment, self-disclosure, and interpersonal aspects of the family environment were satisfactory (Schaefer \& Olson, 1981).

In this self-report questionnaire both partners report their perceptions of actual relationship properties. The major conceptual and methodological issue is whether discrepancies between the two observers are considered to be due to measurement error or to be valid perceptual differences between partners. Secondly, the questionnaire differs from sociological surveys in that it measures not interpersonal events, but subjective experience of psychological interdependence. Schaefer \& Olson (1981) do not provide data on correlation or incongruence between partners nor do they correlate results with measures of behavioural interdependence for intimacy. Research workers who believe that discrepancy in reports represents measurement error may sum or average the reports into a couple score. Research workers who believe that discrepancy is real and valid will investigate incongruency scores. However, the data from both partners do allow some assessment for any systematic response bias, such as conventionality or social desirability, which are absent in the sociological surveys. The PAIR has not been used in epidemiological studies of the relationship of intimacy to the prevalence of emotional illness.

\section{THE MEASUREMENT OF MARITAL INTIMACY}

Waring et al. (1981 b) developed an operational definition of intimacy in marriage, where intimacy is the dimension which most determines marital adjustment (Waring et al. 1980). Marital intimacy has been operationally defined as a multifaceted dimension composed of: affection, commitment, compatibility, expressiveness, identity, sexuality, conflict resolution, and autonomy (Waring et al. 1980, 1981 a). A structured interview, the Victoria Hospital Intimacy Interview (VHII), and a self-report questionnaire, the Waring Intimacy Questionnaire (WIQ), were developed from the operational definition (Waring et al. 1981 a; Waring \& Reddon, 1983).

The WIQ contains 40 items with item efficiency index correlations greater than 0.5 with the overall intimacy score. This procedure is designed to yield minimally redundant scales and a more restrictive use of relevant dimensions which predict intimacy. The test-retest reliability of these 40 items over a two-week interval is 0.89 for males and 0.86 for females and Kuder-Richardson formula-20 reliability is 0.78 for males and 0.81 for females. A highly significant correlation with the PAIR has been demonstrated (Waring \& Reddon, 1983).

Since the issues of reliability and validity for the WIQ are essentially the same as those for the PAIR, the structured interview will be discussed in more detail. The VHII was developed on a sample of 24 couples, where one spouse was in out-patient treatment for neurosis, character disorder, psychophysiological disorder, and/or marital maladjustment, and 24 non-clinical couples (a randomly selected volunteer group) (Waring et al. 1980).

The VHII provides ratings from 1 to 5 on the 8 aspects of a couple's intimacy previously mentioned plus ratings for intimate behaviour during the interview and an overall couple intimacy rating. Thus, a couple's intimacy rating could range from 10 (distant) to 50 (optimal intimacy). The range of scores in this developmental study was $18-48$, with the range of the five raters' scores being \pm 1.5 on any couple. 
The inter-rater reliability between five raters, who trained for over one year, ranged from 0.51 to 0.66 , using weighted $\mathrm{Kappa}\left(\mathrm{K}_{w}\right)$ on the 10 dimensions over 20 interviews, and from 0.68 to 0.83 for interclass correlation (ICC); all these values were statistically significant $(P<0.05)$. In a subsequent study in the general population, the level of intimacy was positively correlated with marital adjustment and negatively correlated with the presence of non-psychotic emotional illness (Waring et al. 1981 b).

In this method both partners report on his or her personal view of the relationship, but there is also direct observation of any incongruity between their perceptions. Unfortunately, the inter-rater reliabilities on the behavioural dimensions were the lowest. By convention, where partners' perceptions differed, the rater would give the lowest score for that aspect of intimacy. This method does have the potential for exploring the accuracy of perceptions as well as actual behaviour in the interview with perceived intimacy in everyday life. However, methodological questions regarding the rather low values for inter-rater reliability limit the general application of the structured interview. Further research is necessary to resolve the problem of rating differences in perception between spouses and which specific facets of behaviour in interviews are predictive of intimate relationships.

\section{DISCUSSION}

The conceptual and methodological issues in studying close relationships where the dyadic relationship is the unit of analysis are far from resolved (Thompsen \& Walker, 1982). All methods of measuring intimacy have their advantages and disadvantages. Boals et al. (1982) recently studied the reliability, validity and utility of three data modes in assessing marital relationships. Using interviews, questionnaires, and two analogue situations, they found high convergent validity across the three data modes and a modest, but significant, superiority of the interview over the other procedures for assessing marital relationships. However, these interviews were conducted with the spouses separately and there were only 12 couples in the study.

One might speculate that a study of convergent validity between sociological surveys, self-report questionnaires, structured interviews and analogue situations for measuring intimacy might replicate Boals et al.'s findings for marital adjustment. Such research might help to resolve questions of conceptual and methodological problems in intimacy research.

Until these questions are answered, research workers should consider increasing the strength of their findings by: (1) increasing the specificity of the study of relationships; (2) allowing for data collection which addresses the theoretical differences between individuals' perceptions and relationship variables; and (3) considering the multifaceted nature of the concept of intimacy in developing operational definitions.

E. M. WARING

\section{REFERENCES}

Aneshensel, C. S. \& Stone, J. D. (1982). Stress and depression: a test of the buffering model of social support. Archives of General Psychiatry 39, 1392-1396.

Beck. A. T. (1963). Thinking and depression. 1. Idiosyncratic content and cognitive distortion. Archives of General Psychiatry 9, 324-333.

Beck, A. T. (1974). Depressive neuroses. In The American Handbook of Psychiatry, Vol. 3 (ed, S. Arieti and Brody), p. 76. Basic Books: New York.

Boals, G. F., Peterson, D. F., Farmer, L., Manny, D. F. \& Robinson, D. L. (1982). The reliability, validity, and utility of three data modes in assessing marital relationships. Journal of Personality Assessment 46 (1), 85-95.

Brown, G. W. \& Harris, T. (1978a). Social Origins of Depression: $A$ Study of Psychiatric Disorder in Women. Tavistock: London.
Brown, G. W. \& Harris, T. (1978 $b$ ). Social origins of depression: a reply. Psychological Medicine 8, 577-588.

Costello, C. G. (1982). Social factors associated with depression: a retrospective community study. Psychological Medicine 12, $329-340$

Dean, D. G. \& Lucas, W. L. (1978). Whose marital adjustment - hers, his or theirs? Psychological Reports 43, 978.

Henderson, S., Byrne, D. G. \& Duncan-Jones, P. (1981). Neurosis and the Social Environment. Academic Press: London.

Hinde, R. A. (1978). Interpersonal relationships - in quest of a science. Psychological Medicine 8, 373-386.

Miller, R. S. \& Lefcourt, H. M. (1982). The assessment of social intimacy. Journal of Personality Assessment 46 (5), 514-518.

Schaefer, M. T. \& Olson, D. H. (1981). Assessing intimacy: the PAIR Inventory. Journal of Marital and Family Therapy 7, 47-60.

Shapiro, M. B. (1979). The social origins of depression by 
G. W. Brown and T. Harris: its methodological philosophy. Behaviour Research and Therapy 17, 597-603.

Solomon, Z. \& Bromet, E. (1982). The role of social factors in affective disorder: an assessment of the vulnerability model of Brown and his colleagues. Psychological Medicine 12, 123-130.

Tennant, C. \& Bebbington, P. (1978). The social causation of depression: a critique of the work of Brown and his colleagues. Psychological Medicine 8, 565-575.

Tennant, C., Bebbington, P. \& Hurry, J. (1982). Social experiences in childhood and adult psychiatric morbidity: a multiple regression analysis. Psychological Medicine 12, 321-328.

Thompsen, L. \& Walker, A. J. (1982). The dyad as the unit of analysis: conceptual and methodological issues. Journal of Marriage and the Family 11, 889-900.

Waring, E. M. (1983). Marital intimacy - a valid concern in medical care. Physician and Patient 11 (2), 68-73.

Waring, E. M. \& Chelune, G. J. (1983). Marital intimacy and self-disclosure. Journal of Clinical Psychology 39 (2), 183-190.
Waring, E. M. \& Patton, D. (1984). Marital intimacy and depression. Presented at the VIIth World Congress of Psychiatry, Vienna, Austria, 1983. Submitted to British Journal of Psychiatry.

Waring, E. M. \& Reddon, J. R. (1983). The measurement of intimacy in marriage: the Waring Intimacy Questionnaire. Journal of Clinical Psychology 39 (1), 53-57.

Waring, E. M., Tillmann, M. P., Frelick, L., Russell, L. \& Weisz, G. (1980). Concepts of intimacy in the general population. Journal of Nervous and Mental Disease 168 (8), 471-474.

Waring, E. M., McElrath, D., Lefcoe, D. \& Weisz, G. (1981a). Dimensions of intimacy in marriage. Psychiatry 4 (2), 169-175.

Waring, E. M., McElrath, D., Mitchell, P. \& Derry, M. E. (1981 b). Intimacy and emotional illness in the general population. Canadian Psychiatric Association Journal 26, 167-172.

Waring, E. M., Reddon, J. R., Corvinelli, M., Chalmers, W. S. \& Vander Laan, R. (1983). Marital intimacy and mood states in a non-clinical sample. Journal of Psychology 115, 263-273. 